\title{
Technology Challenges of Virtual Worlds in Education \& Training - Research directions
}

\author{
Leonel Morgado \\ INESC TEC (formerly INESC Porto) \\ UTAD - University of Trás-os-Montes e Alto Douro \\ Vila Real, Portugal \\ leonelm@utad.pt
}

\begin{abstract}
Research on virtual world use in education and training has focused on the current state of these technologies. In this paper, an overview of the current technological challenges that are faced in this context is provided, along with research directions towards the development of more advanced systems which can render virtual world use more widespread in education and training contexts.
\end{abstract}

Keywords - virtual worlds, education, training, deployment, integration, widespread use

\section{INTRODUCTION}

Research on the educational use of virtual worlds is mostly focused on educational know-how: how actors in the educational process can employ these technologies. A significant body of literature has been developing since the early 1980s, when text-only virtual worlds started being used for this purpose [1], until present-day 3D virtual worlds (Hew $\&$ Cheung [2] and Sara de Freitas [3] provide nice overviews of current knowledge in this regard).

However, studies focusing on educational practices tend to use a static view of technology - as a product used "as is" or designed and developed for a specific purpose. In such studies, the technological perspective is focused merely on the identification of limitations or restrictions imposed by the features of the specific virtual world being used (e.g., [4]; [5]).

However, from a technological viewpoint, virtual worlds are not mere static facts, "as is" products or neutral tools merely streamlining foreseen tasks: rather, they are artefacts, in the sense that they reflect the result of development processes as concrete renderings of knowledge, and as any novel artefacts, by coming into existence they generate new knowledge and processes, changing prior assumptions, and leading to new fields of inquiry and practice. Under this perspective, this paper proposes three lines of inquiry towards an updated understanding of the technological challenges that virtual worlds face when applied to education and training, as well as research directions to overcome those challenges:

- the matter of making the technology available to educational actors;

- the matter of content production, in support of advanced interactions beyond plain dialog;

- the matter of large scale deployment of virtual world technologies, by integration in current information systems, enabling them to be used regularly and commonly by educational actors in general, not just occasionally or only by those with more enthusiasm.

\section{A WORKING DEFINITION OF VIRTUAL WORLDS}

Before presenting the lines of inquiry proper, I wish to clarify the scope: what products or computing systems are understood as "virtual worlds" in the current context? To answer this question, the working definition herewith is based on a usefulness criterion: it aims to identify systems which are not better analysed under a different set of theories.

In the present context of virtual worlds for education and training (E\&T), one might think of virtual worlds as simulations of reality, enriching educational contexts. But this concept does not hold itself autonomously; long have simulations and simulators been used for E\&T, including in the pre-computing era. Research on simulators and simulations is a rich and deep field, from which many virtual worlds' uses can benefit, but this does not wholly encompass virtual worlds as a field. One might think of virtual worlds as games. And indeed many of the best-known virtual worlds are indeed games, such as World of Warcraft or RuneScape. But such a perspective on virtual worlds also does not hold itself autonomously: long has the educational use of videogames been researched. Virtual worlds' uses can benefit from this research, but videogame research does not cover all virtual worlds and encompasses systems which are not virtual worlds. One might think of virtual worlds as social spaces, since social interaction is the focus of many such systems, such as Second Life or Habbo Hotel. But once more, while research literature about social spaces is indeed relevant to the study of virtual worlds, this perspective does not encompass the concept of virtual worlds wholly.

Most authors define "virtual world" using approaches that are either too restrictive or too simple. For instance, the wellknown definition by Castronova [6] requires virtual worlds to have three features: multiuser interactivity, first-person simulation of a physical environment, and persistence of the space, regardless of user presence. While adequate for his economic research perspective, this definition is too restrictive for E\&T use: one needs to go no further than to consider a virtual laboratory of classroom that is created automatically before a session and destroyed afterwards, to have in hands a meaningful E\&T use that is outside Castronova's definition. The other extreme is, for instance, the definition by the IEEE Virtual Worlds Standard Working Group [7]: "A synthetic 
environment consisting of virtual spaces containing objects, inhabitants, and their relationships, which exists in virtuallydefined time." It's such a broad definition that this IEEE working group needs to clarify it as "the term today has become largely synonymous with interactive $3 \mathrm{D}$ virtual environments, where the users take the form of avatars visible to others graphically" - clearly demonstrating that the definition lacks specificity.

Among these extremes, I've put forward in 2009 the proposal that for E\&T purposes, there are only two critical features identifying a virtual world: multiuser interactivity and a physical environment where the use is an immersed avatar [8] (Portuguese-language paper; an English-language version of its arguments was published in 2010 [9]). It's inspired in Castronova's definition, but drops the persistence requirement and clarifies the relationship of the user's avatar with the physical environment, avoiding the visual-inclined "firstperson" term. Under this working definition, virtual worlds include social environments such as Second Life, and games such as World of Warcraft, but also old multiuser games, such SPACEWAR!, one of the earliest videogames [10], and even text-only environments, such as the Multi-User Dungeon environments, created from 1979 onward [11].

\section{TECHNOLOGY CHALLENGES OF VIRTUAL WORLDS IN EDUCATION \& TRAINING}

\section{A. Accessing virtual worlds}

In some cases, multiuser interaction is provided via multiple interfaces connected to the same system (i.e., the aforementioned SPACEWAR! [10], many current console games, and some online games which combine keyboard sections for each player). But for many systems, multiuser interaction is achieved via computer networking. Further, the concept of computational devices is evolving, and one must consider not only traditional desktops, but also smartphones, tablets, and novel augmented reality wearables. Thus, remote access issues take on a major role in virtual world access challenges. In this paper, the three following sub-challenges are reflected upon:

\section{1) Network architectures and features \\ 2) User-end software \\ 3) Isolation vs. interconnection}

The first of these dimensions includes aspects such as architectures such as client-server or peer-to-peer (P2P). The first of these is widespread, but lesser known systems employ P2P architectures: Open Cobalt is possibly the only operational system of this ilk, but other modules or proposals exist, such as Walkad [13] and Badumna [14]. E\&T consequences of adopting virtual worlds based on these confronting alternatives need to be studied. For instance, by analysing which E\&T scenarios would benefit from the decentralized storage and virtual world execution provided by $\mathrm{P} 2 \mathrm{P}$, and which scenarios would be rendered unviable by it; which educational management methods for teachers, trainers, and educational organizations would these alternative network architectures imply; and many more. But network behaviour of virtual worlds also requires much research. There are scarce studies in this regard (e.g., [15]), and most were conducted with early systems that supported only a few users. Knowledge of network behaviour of virtual worlds is important for wider adoption of these platforms, since organizations are currently unable to estimate which network configurations and user settings enable specific activities, particularly in variable bandwidth or intermittent connectivity situations, in combination with varied ecologies of other networked applications, and other concerns of the modern networking environment.

The second dimension looks into whether virtual worlds are accessed via specific software installed locally on a user computing device or through more generic interfaces, such as Web browsers. Obviously, all systems such as those mentioned in the opening paragraph of this section employ locally-installed software, but so do many network-based systems, such as Second Life and World of Warcraft, which rely on locally-installed clients for communicating with virtual world servers. Having locally-installed software places various challenges to virtual world adoption for E\&T: it requires that users have administrative access to their machines (common in home computers; uncommon in mobile devices and in organizations); it implies the risk of software conflicts or hardware shortcomings (for instance, inadequate graphics drivers are a common issue); it involves running networkaware applications whose security shortcomings are not well known. While none of these issues is critical for individual uses, any attempt at widespread use (or business-critical use) of virtual worlds will run face-flat on them. Research is needed on how to streamline installation and updating of virtual world client software, how to manage, monitor, track, and debug client software, and on security vulnerabilities and attack vectors. On the other hand, the use of generic interfaces is still in its infancy. Some virtual world exist which are based on Web browsers, but if they require the installation of specific plug-ins, the aforementioned issues remain. Some do employ generic or widespread plug-ins, such as Adobe Flash (e.g., FarmVille, MyVineyard), and others on the WebGL specifications, which have the potential to be a widespread standards (but currently is only supported for a few browsers: Chrome, Opera (announced), Blackberry browser, Opera Mobile, Firefox for Android. No full support is as yet announced for Internet Explorer, Firefox, or Safari [16]. There is a lack of research on operational behaviour of virtual worlds in these platforms, on usability, interfaces, and vulnerabilities. Finally, another incipient approach is providing virtual worlds via video streaming, something some companies such as OnLive, OTOY, and Gaikai already do for games. Which E\&T uses of virtual worlds can be enabled by the operational constraints of this approach? Which are not viable? Research on this regard is inexistent.

The third dimension, of isolation vs. interconnection, impacts the scope of viable E\&T activities. Each educational context includes actors and resources. The most common situation is for both to be restricted to a specific server or server grid, managed by a single entity. This prevents users from visiting other virtual worlds without losing their identity and prevents the sharing of virtual resources [17]. Sometimes, not even within the same virtual world: some virtual world are 
split among parallel copies of themselves, distinct and disjoint, with different sets of users and resources - a load-balancing approach known as sharding, quite common in commercial virtual gaming worlds. This is quite analogous to the status of online fora in the Bulletin Board Services era, prior to the current World Wide Web, a analogy point out before [18]. A specific technology, OpenSimulator, has a network interconnection module, called Hypergrid, which enables different organizations to share users and resources across virtual worlds, but this has severe scaling and security risks [19], and remains locked within a specific server technology. Other proposals exist, which are server-agnostic, but remain in the realm of early research proposals (e.g., [20][21]), and much effort is required in this regard.

\section{B. Content production in/for virtual worlds}

After educational actors have access to a virtual world, what do they find there? What's the content, from whence does it come? In a virtual world the user experiences a virtual shared space, with its own features and content, such as topography, objects, and agents (both controlled by other users or by computer systems). This content provides context and features for the educational process. Thus, this second dimension centres around content production. At this level, the most relevant distinction from the perspective of the educational process is the origin of content:

\section{1) Produced by experts \\ 2) Produced by actors of the educational process}

The relevance of this distinction arises from the production methods associated with these kinds of content. Depending on whether production is done by experts in the various technologies related to virtual worlds (i.e., modellers, artists, programmers, etc.), or by the actors of the educational process themselves, there will be consequences for the feasibility of two kinds of educational use of computing: the kind where it entirely determines the context and the acts arising from its use; and the kind where computing is only a tool in support in educational actions.

For the first case, with content production at the hands of experts, the flexibility of planning and execution of educational actions is diminished. It may happen that in some cases actors of the educational process are able to fully determine how that content production takes place (e.g., if they are part of the design \& development process, or if they have regular access to technical experts). But even in those cases, the management of human resources for production implies some restraint in the planning or development of production. But even for experts, there is a general lack of research on the implications of adopting different tools over others, on the workload and risk management associated with a specific path of development, and on the flexibility of subsequent changes and updates (Anderson provides one of the scarce papers on these matters [22]).

For the second case, with content production accessible to the actors of the educational process, there is greater flexibility in the planning and execution of educational actions, since it may be feasible to change the content (or create new content) in response to specific situations that occur; or even plan and develop an educational process which includes in its design those very acts of content production by its actors. In virtual worlds developed for specific purposes, user-editing options are typically quite limited, often just at the level of some avatar customizations and spatial placement of predefined items. But in virtual worlds meant for cooperation, that is, virtual worlds designed as platforms, the range of possibilities is quite large. Such are the cases of Activeworlds, IMVU, Open Cobalt, OpenSimulator, OpenWonderland, Second Life, and Twinity, among others. Research is needed on the enduser experience and development processes, on the adequacy of tools, on training for use of those tools, and on improving content developing tools from their current early state. Particularly worth of research is going beyond the development of 3D models and into the development of animations and behaviours. Rather than simply provide simpler versions of professional tools, the vast research background on programming by demonstration [23] and computer-supported cooperative work [24] should be tapped, in order for users to demonstrate animations and behaviours and generalize from them, not only alone but in teams.

\section{Deployment of virtual worlds}

A third dimension is the integration of virtual worlds with other education \& training systems. This revolves around large scale deployment of the use of these technologies, to render viable its regular and prolonged use by the educational actors in general - not just occasionally or by enthusiasts. The motivation for this concern arises from typical contexts in education and training, where teaching needs to take place in actual courses, attended by dozens or hundreds of students/trainees: for instance how can assignments be tracked readily regarding their development and progress, so teachers/trainers have a clear perspective of possible learning support needs or tutoring, or for providing extra resources? How can existing learning support systems collect data from virtual worlds for assessment and communicate with them to provide feedback and guidance to learners? If virtual worlds are detached from management platforms, this requires 3D world actions for management of non-3D data.

There have been efforts to overcome this, such as the wellknown SLOODLE project [25], offering Moodle access from Second Life. Or the BbSL project [26], offering some linking between Second Life and Blackboard. There are also some efforts for controlling Second Life and OpenSimulator from Web-based systems [27][28]. Overall two major approaches emerged:

- under the SLOODLE/BbSL approach [25] [26], the external system is seen as a repository or source of information, and the virtual world as a view or interface for them;

- $\quad$ under an alternative approach, which is pursued at several institutions [27][28][29], the virtual world is seen as a space where activities take place under management and support of the external system.

A key distinction between these approaches is that they arise from different perspectives of use and deployment. The first of these approaches was born out of a survey of 
individual teachers which were using the Second Life virtual world [25]. This leads towards contexts which are fundamentally established by the actors in charge of execution the educational actions, typically with high degrees of autonomy: teachers using this virtual world in support of ongoing activities and with the freedom to plan and control the educational process. Thus, an approach focused on individuals' needs, not on organizational needs. The second approach was born out of the desire to integrate virtual world activities in existing administrative and organizational processes: regardless of whether activities take place in a virtual world or not, their encompassing organization will have specific requirements, such as attendance records or privacy controls [5]. Thus, an approach focused on organizational needs, not on individuals' needs.

Both approaches (individuals and organizations) are complementary, so both provide innovative concepts and perspectives for integrating virtual worlds in external systems. But they establish distinct sets of requirements, and consequently reach distinct architectures and solutions. Research is needed to ascertain these requirements clearly, to test, model, and ultimately develop better versions of these or novel approaches, and to identify training needs to render viable widespread deployment of virtual worlds.

\section{FINAL THOUGHTS}

As a final reflection, virtual worlds have come a long way in E\&T since their text-only early days throughout the 1980s. The current breed of graphics-intensive virtual worlds is widespread in the gaming field, and enjoying significant know-how and research in the educational field. However, the research in this field is not looking at the underlying technological issues, and this is probably hindering the possibilities of more widespread use of virtual worlds in educational and training contexts.

In this paper, an encompassing view of the technological challenges facing virtual worlds was provided from the perspective of their use for education and training. Hopefully this overview will contributing for the focusing of research efforts towards a newer batch of virtual world technologies, more adequate for widespread use in education.

\section{ACKNOWLEDGMENT}

This work is funded (or part-funded) by the ERDF European Regional Development Fund through the COMPETE Programme (operational programme for competitiveness) and by National Funds through the FCT Fundação para a Ciência e a Tecnologia (Portuguese Foundation for Science and Technology) within project «FCOMP - 01-0124-FEDER-022701.

\section{REFERENCES}

[1] T L Fanderclai, "MUDs in Education: New Environments, New Pedagogies", Computer-Mediated Communication Magazine, vol. 2, no. 1,1995 , p. 8 .

[2] K F Hew and W S Cheung, "Use of three-dimensional (3-D) immersive virtual worlds in K-12 and higher education settings: A review of the research", British Journal of Educational Technology, vol 41, no. 1, 2010, pp. 33-55.

[3] S de Freitas, "Serious virtual worlds: A scoping study", 2008. Bristol, UK: Joint Information Systems Committee.

[4] M D Dickey, "Brave new (interactive) worlds: A review of the design affordances and constraints of two 3D virtual worlds as interactive learning environments". Interactive Learning Environments, vol. 13, no. 1, 2005, pp. 121-137.

[5] A Vilela, M Cardoso, D Martins, A Santos, L Moreira, H Paredes, P Martins, and L Morgado, "Privacy challenges and methods for virtual classrooms in Second Life Grid and OpenSimulator", in 2010 Second International Conference on Games and Virtual Worlds for Serious Applications (VS-GAMES), 2010, pp. 167-174. Washington, DC, USA: IEEE Computer Society.

[6] E Castronova, "Virtual Worlds: A First-Hand Account of Market and Society on the Cyberian Frontier", The Gruter Institute Working Papers on Law, Economics, and Evolutionary Biology, vol. 2, no. 1, 2001.

[7] IEEE VW Standard Working Group, IEEE VW Standard Working Group [on-line], http://www.metaversestandards.org/ (2013-06-20).

[8] L Morgado, "Os mundos virtuais e o ensino-aprendizagem de procedimentos", Educação \& Cultura Contemporânea, vol. 13, no. 6, 2009, pp. 35-48.

[9] L Morgado, J Varajão, D Coelho, C Rodrigues, C Sancin, V Castello, "The Attributes and Advantages of Virtual Worlds for Real World Training", The Journal of Virtual Worlds and Education, vol. 1, no. 1, 2010, pp. 15-35.

[10] J M Graetz, "SPACEWAR: Real-Time Capability of the PDP-1", in "DECUS Proceedings 1962 - Papers and Presentations of The Digital Equipment Computer Users Society, Maynard, Massachusetts", 1963, pp. 37-39. Maynard, MA, USA: Digital Equipment Computer Users Society.

[11] R Bartle, "Early MUD history". E-mail message sent on November 15 1990, at 19h00:55 GMT. Available online at http://www.linnaean.org/ lpb/muddex/bartle.txt (2013-06-20).

[12] Duke University, "Open Cobalt Website". http://www.opencobalt.org/ (2013-06-20).

[13] M Varvello, C Diot, E Biersack, "A Walkable Kademlia Network for Virtual Worlds", in "Proceedings of the 28th IEEE international conference on Computer Communications Workshops", 2009, pp.313314. Piscataway, NJ, USA: IEEE Press.

[14] S Kulkarni, S Douglas, and D Churchill, "Badumna: A decentralised network engine for virtual environments", Computer Networks: The International Journal of Computer and Telecommunications Networking, vol. 54, no. 12 (2010), pp. 1953-1967.

[15] M Ferreira, R Morla, "Second life in-world action traffic modeling", in NOSSDAV ' 10 - Proceedings of the 20th international workshop on Network and operating systems support for digital audio and video, 2010 , pp. 3-8. New York, NY, USA: ACM.

[16] Anon., "Can I Use WebGL”, http://caniuse.com/webgl (2013-06-20).

[17] G Morgan, "Challenges of Online Game Development: A Review", Simulation \& Gaming, vol. 40, no. 5, 2009, pp. 688-710.

[18] L Morgado, "Interconnecting virtual worlds", Journal of Virtual Worlds Research, vol. 3, no. 1, 2009, pp. 4-7.

[19] J Clark-Casey, "Scaling OpenSimulator: An Examination of Possible Architectures for an Internet-Scale Virtual Environment Network", Master Thesis. Oxford, UK: Kellogg College, University of Oxford.

[20] B Bossche, B Vleeschauwer, T Verdickt, F Turck, B Dhoedt, and P Demeester, "Autonomic microcell assignment in massively distributed online virtual environments", Journal of Network and Computer Applications, vol. 32, no. 6, 2009, pp. 1242-1256.

[21] C Ghosh, RP Wiegand, B Goldiez, T Dere, “An architecture supporting large scale MMOGs", in "SIMUTools '10 - Proceedings of the 3rd International ICST Conference on Simulation Tools and Techniques", 2010. Brussels, Belgium: ICST.

[22] E F Anderson, "A Classification of Scripting Systems for Entertainment and Serious Computer Games", in 2011 Third International Conference on Games and Virtual Worlds for Serious Applications (VS-GAMES), 2011, pp. 47-54. 
[23] H Lieberman, "Your Wish is My Command: Programming By Example", 2001, San Francisco, CA, USA: Morgan Kaufmann.

[24] A Cruz, A Correia, H Paredes, B Fonseca, L Morgado, P Martins, "Towards an overarching classification model of $\mathrm{CSCW}$ and groupware: a socio-technical perspective", in "Collaboration and Technology - 18th International Conference CRIWG 2012, Raesfeld, Germany, September 16-19 2012, Proceedings", 2012, pp. 41-56. Berlin: Springer-Verlag.

[25] J Kemp and D Livingstone, "Putting a Second Life 'Metaverse' Skin on Learning Management Systems", in Kemp, J. \& Livingstone, D. (Eds.) Proceedings of the First Second Life Education Workshop, Part of the 2006 Second Life Community Convention, August 18th-20th 2006, Fort Mason Centre, San Francisco, Ca., p.13-18, 2006. Paisley, Scotland, UK: The University of Paisley.

[26] OSCELOT, "Blackboard - Second Life Integration", http://projects.oscelot.org/gf/project/bbslintegration/wiki/ (2013-06-20).

[27] B Fonseca, H Paredes, J Rafael, L Morgado, P Martins, "A Software Architecture for Collaborative Training in Virtual Worlds: F-16 airplane engine maintenance", in Adriana S. Vivacqua, Carl Gutwin and Marcos R.S. Borges (Eds.) "Collaboration and Technology: 17th International Conference, CRIWG 2011, Paraty, Brazil, October 2-7, 2011. Proceedings", 2011, pp. 102-109. Berlin, Germany: Springer.

[28] E Silva, N Silva, H Paredes, P Martins, B Fonseca, L Morgado, "Development of platform-independent multi-user choreographies for virtual worlds based on ontology combination and mapping", in Erwig, M.; Stapleton, G.; Costagliona, G. (Eds.), "VL/HCC 2012, Proceedings of the 2012 IEEE Symposium on Visual Languages and Human-Centric Computing, September 30-October4 - Innsbruck, Austria", 2012, pp. 149-152. Los Alamitos, CA, USA: IEEE Computer Society.

[29] J Maderer, C Gütl, M Al-Smadi, "Formative Assessment in Immersive Environments: A Semantic Approach to Automated Evaluation of User Behavior in Open Wonderland", in iED 2013 Proceedings, 2013 